\title{
Caracterização das internações por intoxicação medicamentosa, São Paulo, 2004 a 2006
}

\author{
Characterization of admissions due to medicine poisoning, São Paulo, \\ 2004 to 2006
}

\section{Caracterización de las internaciones por intoxicación medicamentosa,} São Paulo, 2004 a 2006

Janessa de Fátima Morgado de OLIVEIRA ${ }^{1}$ Gabriela Arantes WAGNER ${ }^{2}$

Nicolina Silvana ROMANO-LIEBER ${ }^{3}$

José Leopoldo Ferreira ANTUNES ${ }^{4}$

${ }^{1}$ Faculdade de Saúde Pública da Universidade de São Paulo (FSP-USP) 01246-904, São Paulo - SP, Brasil ${ }^{2}$ Departamento de Medicina Preventiva, Universidade Federal de São Paulo (UNIFESP) 04223-062 São Paulo - SP, Brasil ${ }^{3}$ Departamento de Política, Gestão e Saúde. Faculdade de Saúde Pública da Universidade de São Paulo (FSP-USP) 01246-904, São Paulo - SP, Brasil

${ }^{4}$ Departamento de Epidemiologia. Faculdade de Saúde Pública da Universidade de São Paulo (FSP-USP) 01246-904, São Paulo, SP, Brasil

\section{Resumo}

Introdução: Os medicamentos representam um instrumento essencial para a capacidade resolutiva dos serviços de saúde por estarem associados a finalidades profiláticas, curativas, paliativas ou diagnósticas, mas sua grande utilização favorece o surgimento de problemas e riscos. As intoxicações são um exemplo e constituem um problema de saúde pública no país. Objetivo: As internações por intoxicação medicamentosa no município de São Paulo entre os anos 2004 e 2006 foram caracterizadas quanto à frequência em relação ao total de casos por faixa etária, sexo, tempo de internação, intenção e medicamentos envolvidos. Material e métodos: Os coeficientes de internação foram calculados segundo faixa etária e sexo. Resultados: $\mathrm{O}$ tempo total de internação para os casos estudados foi de 14.852 dias (média de 4,4 dias). A idade média dos internados foi de 33,5 anos, com maior frequência para mulheres $(60,5 \%)$. Houve maior prevalência de intoxicações por benzodiazepínicos $(7,5 \%)$ e antibióticos sistêmicos não especificados $(7 \%)$. Os maiores coeficientes de internação foram observados entre idosos ( $\geq 70$ anos) e sexo feminino em todos os anos de estudo. Conclusão: São necessárias ações para diminuição do número de casos de intoxicações entre idosos e mulheres (alta prevalência do uso de medicamentos), e para os jovens (tentativas de suicídio).

Descritores: Admissão do Paciente; Hospitalização; Envenenamento; Preparações Farmacêuticas.

\section{Abstract}

Introduction: Medicines represent an essential tool for the health services' ability to solve health problems, being used with prophylactic, curative, palliative or diagnostic purposes, but their great use is associated to emergence of problems and risks. Poisoning are an example and constitute a public health problem in the country. Objective: Hospital admissions due to medicine poisoning in São Paulo City between 2004 and 2006 were characterized (frequency in relation to the total number of cases) by age group, sex, duration of hospitalization, intention and medicines involved. Methods: The coefficients of hospitalization were calculated according to age group and sex. Results: Total time duration of hospitalizations for the cases studied was 14,852 days (mean of 4.4 days). Mean age of hospitalized patients was 33.5 years, with a higher frequency for women (60.5\%). There was a higher prevalence of benzodiazepine poisonings (7.5\%) and unspecified systemic antibiotics poisonings (7\%). The highest coefficients of hospitalization were observed among the elderly ( $\geq 70$ years) and female in all the years of study. Conclusion: Action is needed to reduce the number of medicine poisoning cases in elderly and women (high prevalence of medicine use), and in young people (suicide attempts).

Descriptors: Patient Admission; Hospitalization; Poisoning; Pharmaceutical Preparations.

\section{Resumen}

Introducción: Los medicamentos representan un instrumento esencial para la capacidad resolutiva de los servicios de salud por estar asociados a fines profilácticos, curativos, paliativos o diagnósticos, pero su gran utilización favorece el surgimiento de problemas y riesgos. Las intoxicaciones son un ejemplo y constituyen un problema de salud pública en el país. Objetivo: Las internaciones por intoxicación medicamentosa en el municipio de São Paulo entre los años 2004 y 2006 fueron caracterizadas en cuanto a la frecuencia con relación al total de casos por edad, sexo, tiempo de internación, intención y medicamentos involucrados. Métodos: Los coeficientes de internación se calcularon según edad y sexo. Resultados: Se verificó que el tiempo total de internación para los casos estudiados fue de 14.852 días (promedio de 4,4 días). La edad media de los internados fue de 33,5 años, con mayor frecuencia para mujeres $(60,5 \%)$. Se observó una mayor prevalencia de intoxicaciones por benzodiazepinas $(7,5 \%)$ y antibióticos sistémicos no especificados (7\%). Los mayores coeficientes de internación se observaron entre los ancianos ( $\geq 70$ años) y sexo femenino en todos los años de estudio. Conclusión: Se necesitan acciones para disminuir el número de casos de intoxicaciones entre ancianos y mujeres (alta prevalencia del uso de medicamentos), y para los jóvenes (intentos de suicidio).

Descriptores: Admisión del Paciente; Hospitalización; Envenenamiento; Preparaciones Farmacéuticas.

\section{INTRODUÇÃO}

Os medicamentos são desenvolvidos com o intuito de beneficiar quem os utiliza ${ }^{1}$. Estão associados a finalidades profiláticas, curativas, paliativas ou diagnósticas ${ }^{2}$. Por isso, são responsáveis por boa parte das despesas com o setor saúde, tanto público quanto privado ${ }^{3-5}$.

No entanto, desde 1994, os medicamentos assumiram a primeira posição dentre as intoxicações para o conjunto de agentes tóxicos estudados, respondendo por $24,5 \%$ dos casos registrados no país ${ }^{2}$. A intoxicação exógena também se encontra entre os três principais meios utilizados nas tentativas de suicídio, sendo que em $70 \%$ dos casos estão relacionados os medicamentos e pesticidas ${ }^{6}$. Esses dados demonstram que os medicamentos podem ser causadores de danos à saúde.
Assim, é pertinente a investigação específica de determinantes de intoxicações medicamentosas agudas para diferentes sexos, faixas etárias, circunstâncias e classes farmacológicas, de forma a favorecer o estabelecimento de metas específicas de prevenção ${ }^{7}$. O estudo teve por objetivo descrever as internações por intoxicação medicamentosa em relação às variáveis sexo, faixa etária, tempo de internação, intenção envolvida e medicamentos, para os casos ocorridos no município de São Paulo, Estado de São Paulo, Brasil, no período de 2004 a 2006.

\section{MATERIAL E MÉTODO}

- Desenho e população de estudo

Trata-se de estudo descritivo transversal. A população 
estudada foi a do município de São Paulo, o que viabilizou o trabalho com o universo das internações pelo Sistema Único de Saúde (SUS) no município.

O estudo foi aprovado pelo Comitê de Ética em Pesquisa da Faculdade de Saúde Pública da Universidade de São Paulo.

- Coleta da informação

Os dados de internação foram disponibilizados pelo Sistema de Informações Hospitalares do SUS (SIH/SUS) para o período de 2004 a 2006. No período de estudo ocorreram 2.062.518 internações no município de São Paulo, sendo 741.096 internações no ano de 2004, 636.192 em 2005 e 685.237 em 2006. Houve predomínio de internações de indivíduos do sexo feminino $(60 \%)$ e da faixa etária de até 36 anos $(50,1 \%)$, sendo que a faixa etária de menores de 5 anos já corresponde a $10,6 \%$ das internações.

Os dados de internação por intoxicação medicamentosa foram obtidos do banco de dados de internações para o município de São Paulo. Foram consideradas internações por intoxicação medicamentosa todo registro que apresentou como diagnóstico principal ou secundário os seguintes códigos da décima revisão da Classificação Nacional de Doenças (CID-10): F11.0, F13.0, F15.0, F16.0, F19.0 (intoxicações agudas em pacientes com transtornos mentais e comportamentais); F55 (intoxicações agudas em pacientes com síndromes comportamentais associadas a fatores físicos); P04.0 (reações e intoxicações decorrentes da administração de opiáceos e tranquilizantes à mãe, durante o trabalho de parto; P93 (reações e intoxicações devido a fármacos administrados ao feto e neonato); T36.0-T36.9; $\quad$ T37.0-T37.9; $\quad$ T38.0-T38.9; $\quad$ T39.0-T39.9; $\mathrm{T} 40.2 ; \mathrm{T} 40.3 ; \mathrm{T} 40.4 ; \mathrm{T} 41.0-\mathrm{T} 41.5 ; \mathrm{T} 42.0-\mathrm{T} 42.8 ; \mathrm{T} 43.0$ T43.9; T44.0-T44.9; T45.0-T45.9; T46.0-T46.9; T47.0T47.9; T48.0-T48.7; T49.0-T49.9; T50.0-T50.9 (intoxicações classificadas segundo agente medicamentoso); X40.0-X40.9; $\mathrm{X} 41.0-\mathrm{X} 41.9 ; \quad \mathrm{X} 42.0-\mathrm{X} 42.9 ; \quad \mathrm{X} 43.0-\mathrm{X} 43.9 ; \quad \mathrm{X} 44.0-\mathrm{X} 44.9$ (intoxicação acidental); X60.0-X60.9; X61.0-X61.9; X62.0X62.9; X63.0-X63.9; X64.0-X64.9 (autointoxicação intencional/ tentativa de suicídio); X85 (agressão causada por fármacos, medicamentos e substâncias biológicas); Y10.0Y10.9; Y11.0-Y11.9; $\quad \mathrm{Y} 12.0-\mathrm{Y} 12.9 ; \quad \mathrm{Y} 13.0-\mathrm{Y} 13.9$ e Y14.0-Y14.9 (intoxicação com intenção não determinada).

\section{- Análise de dados}

Foi estudada a frequência de internação por intoxicação medicamentosa (número observado em relação ao total de casos) segundo faixa etária, sexo, tempo de internação, intenção e medicamentos envolvidos para os pacientes atendidos e residentes no município de São Paulo no período de 2004 a 2006.

A magnitude das internações foi estimada através do cálculo do coeficiente de internações por faixa etária e sexo para cada ano. Para o cálculo foram pesquisados também dados oficiais de população paulistana junto à Fundação IBGE, estimados a cada ano pelo órgão. Para estimar os coeficientes de internação por intoxicação medicamentosa, foi considerada a estratificação disponibilizada para os dados de população para o município: menores de 5 anos; 5 a 9 anos; 10 a 14 anos; 15 a 19 anos; 20 a 29 anos; 30 a 39 anos; 40 a 49 anos; 50 a 59 anos; 60 a 69 anos; idade superior a 70 anos.

$\mathrm{O}$ cálculo das frequências e dos coeficientes de internação foi realizado no programa Excel®2013

\section{RESULTADOS}

Das 2.062.518 internações que ocorreram no período de estudo, $3.366(0,16 \%)$ foram selecionados por se tratarem de internações por intoxicação medicamentosa. Para cada 10.000 internações, 16 tiveram diagnóstico primário ou secundário de intoxicação medicamentosa no período.

Dos casos de internações por intoxicação medicamentosa, 1.078 ocorreram em 2004, 1.013 em 2005 e
1.275 em 2006. Das 3.366 internações estudadas, 2.843 internações eram provenientes da rede SUS (Autorizações de Internações Hospitalares - 84,5\% AIH) e 523 internações eram provenientes da rede suplementar (Comunicações de Internações Hospitalares - 15,5\% CIH).

O tempo total para as internações foi de 14.852 dias, sendo que 3.158 internações tiveram entre 1 e 365 dias. A data de internação e saída é a mesma em 157 casos, não há data de saída em 49 casos e a data de saída antecede a de entrada em 2 casos. $O$ tempo médio de internação foi de 4,4 dias, sendo que $69,9 \%$ dos casos tiveram de 1 a 4 dias de internação.

A idade média das pessoas internadas foi de 33,5 anos, variando de menos de 1 ano a 97 anos. Os casos foram mais frequentes entre os indivíduos de 20 a 29 anos (21,6\%), sendo que $49,8 \%$ deles ocorreram para os indivíduos com 20 a 49 anos. Os jovens de 15 a 19 anos e as crianças de 1 a 4 anos também se destacaram, correspondendo a $9,1 \%$ e $7,6 \%$ dos casos, respectivamente.

Verificou-se um total de 2.035 internações para o sexo feminino (60,5\% dos casos estudados). Os coeficientes de internação para o sexo feminino também foram maiores para os anos estudados, conforme pode ser observado nas Figuras 1,2 e 3.

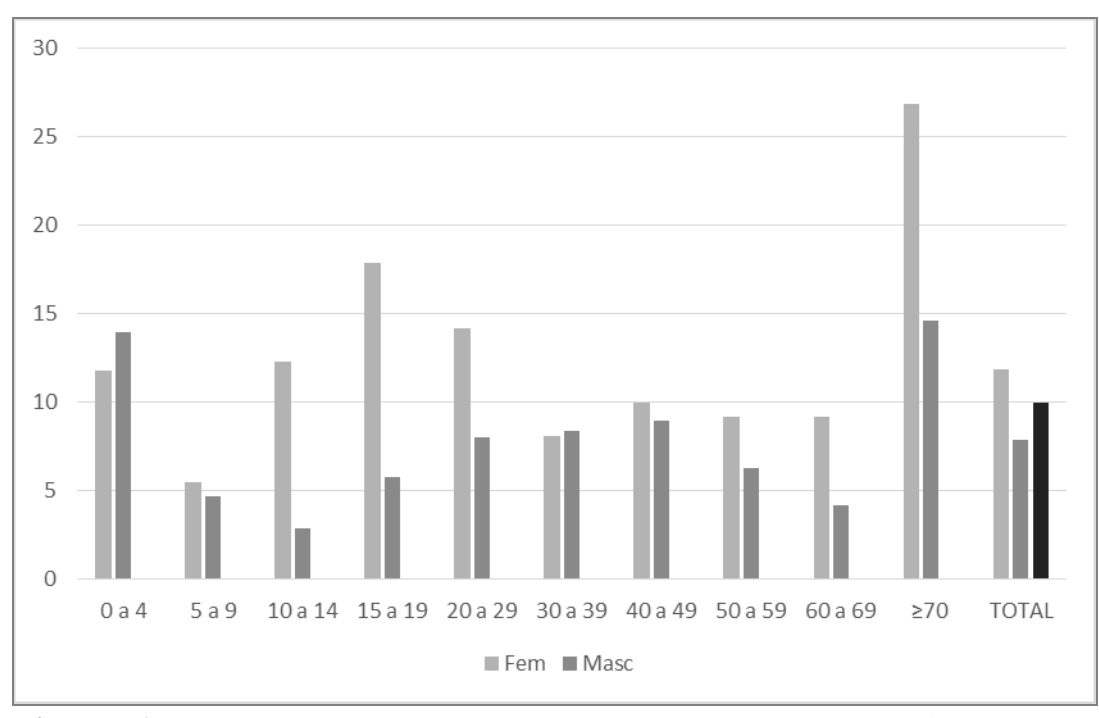

Figura 1: Internações por intoxicação medicamentosa: coeficientes de internação para 100.000 habitantes. Município de São Paulo, 2004.

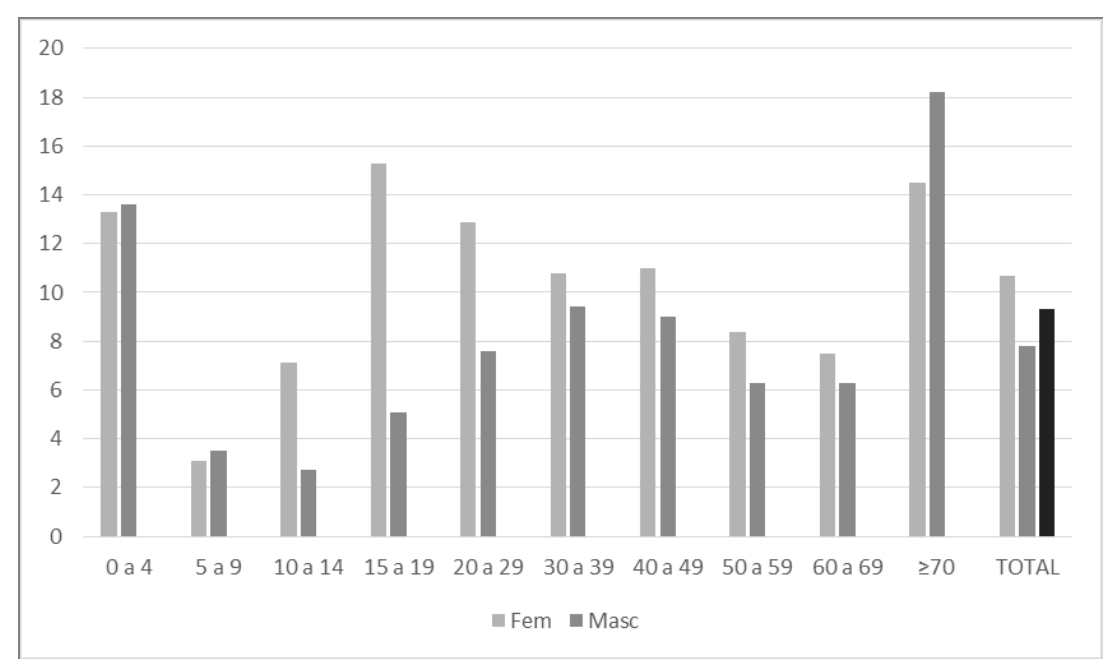

Figura 2: Internações por intoxicação medicamentosa: coeficientes de internação para 100.000 habitantes. Município de São Paulo, 2005.

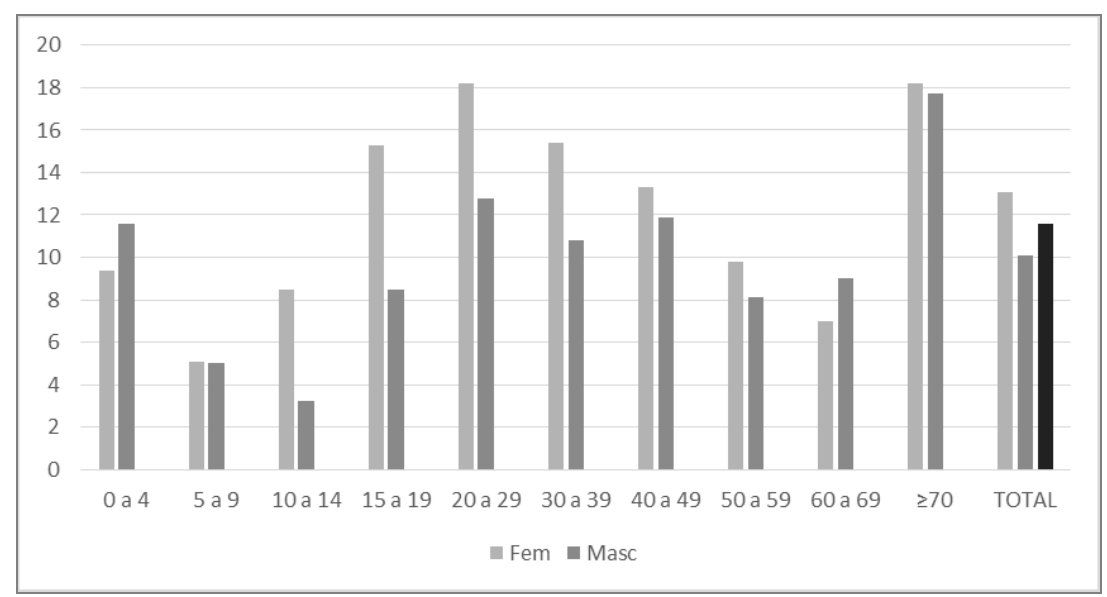

Figura 3: Internações por intoxicação medicamentosa: coeficientes de internação para 100.000 habitantes. Município de São Paulo, 2006. 
Esse padrão predominou entre as faixas etárias, com exceção da faixa etária de 0 a 4 anos para todos os anos estudados; das faixas de 5 a 9 anos e de 70 anos e mais para 2005; e da faixa etária de 60 a 69 anos para o ano de 2006, para as quais os coeficientes de internação por intoxicação medicamentosa foram maiores para o sexo masculino (Figuras 1, 2 e 3).

Ainda nas Figuras 1, 2 e 3, observa-se os maiores coeficientes para a faixa etária dos mais idosos (idade igual ou superior a 70 anos). No ano de 2006, o coeficiente de internação por intoxicação medicamentosa é semelhante para esta faixa etária e a faixa de 20 a 29 anos para o sexo feminino. Verifica-se que os coeficientes aumentaram para as faixas etárias 20 a 29 anos, 30 a 39 anos (principalmente para o sexo feminino), 40 a 49 anos (para ambos os sexos) no período de estudo, e 50 a 59 anos entre 2005 e 2006 (para ambos os sexos).

As internações foram caracterizadas quanto ao agente envolvido. Os diagnósticos principais mais frequentes relacionados ao tempo de internação (correspondentes a $77,5 \%$ dos casos estudados) são apresentados na Tabela 1. Verifica-se pela tabela que os principais agentes envolvidos nas internações por intoxicação medicamentosa, segundo os tempos de duração da internação mais frequentes para o período de estudo, foram outras drogas, medicamentos e substâncias biológicas e as não especificadas (T50.9), que correspondem a $17,5 \%$ das internações; benzodiazepínicos (T42.4), que correspondem a 7,5 \% das internações; e antibióticos sistêmicos não especificados (T36.9), que correspondem a 7\% das internações.

Tabela 1. Internações por intoxicação medicamentosa: diagnósticos principais mais frequentes (\%), segundo tempo de internação município de São Paulo (2004-2006).

\begin{tabular}{|c|c|c|c|c|c|c|}
\hline \multirow{2}{*}{$\begin{array}{c}\text { Diagnóstico } \\
\text { Primário }\end{array}$} & \multicolumn{5}{|c|}{ Tempo de Internação (dias) } & \multirow{2}{*}{$\begin{array}{c}\text { Total } \\
\text { por } \\
\text { Código }\end{array}$} \\
\hline & 1 & 2 & 3 & 4 & 11 a 30 & \\
\hline $\mathrm{F}$ & 4,5 & 3,2 & 4,6 & 2,0 & 13,2 & 4,8 \\
\hline T36.9 & 7,7 & 7,2 & 7,1 & 6,1 & 3,9 & 7,0 \\
\hline T39.0 & 1,1 & 1,6 & 0 & 0 & 0 & 0,9 \\
\hline $\mathrm{T} 42.1$ & 3,8 & 3,8 & 5,7 & 4,5 & 4,7 & 4,2 \\
\hline $\mathrm{T} 42.3$ & 3,2 & 3,8 & 3,8 & 3,5 & 4,3 & 3,6 \\
\hline $\mathrm{T} 42.4$ & 10,2 & 7,7 & 5,2 & 4,0 & 2,7 & 7,5 \\
\hline T42.6 & 1,3 & 2,0 & 0 & 0 & 2,7 & 1,4 \\
\hline $\mathrm{T} 42.7$ & 1,3 & 0 & 0 & 0 & 0 & 0,5 \\
\hline T43.0 & 3,2 & 2,5 & 3,8 & 0 & 2,7 & 2,8 \\
\hline $\mathrm{T} 43.2$ & 2,6 & 3,2 & 0 & 0 & 2,7 & 2,2 \\
\hline $\mathrm{T} 43.4$ & 1,2 & 0 & 0 & 0 & 0 & 0,5 \\
\hline T43.5 & 1,3 & 0 & 0 & 0 & 0 & 0,5 \\
\hline $\mathrm{T} 43.8$ & 1,5 & 2,1 & 0 & 0 & 0 & 1,2 \\
\hline T43.9 & 3,0 & 3,4 & 3,8 & 4,0 & 3,1 & 3,3 \\
\hline $\mathrm{T} 45.5$ & 1,5 & 0 & 0 & 0 & 0 & 0,6 \\
\hline $\mathrm{T} 46.0$ & 0 & 0 & 0 & 0 & 3,5 & 0,3 \\
\hline $\mathrm{T} 47$ & 0 & 0 & 0 & 0 & 0 & 0 \\
\hline $\mathrm{T} 50$ & 2,4 & 0 & 0 & 0 & 0 & 0,9 \\
\hline T50.9 & 15,6 & 19,4 & 18,8 & 21,7 & 13,6 & 17,5 \\
\hline
\end{tabular}

*Fonte: Sistema de Informações Hospitalares

**Nota:

Códigos da CID-10 escolhidos para o estudo - F: intoxicações agudas em pacientes com ranstornos mentais e comportamentais; T36.9: antibióticos sistêmicos não especificados; T39.0: salicilatos; T42.1: iminostilbenos - carbamazepina; T42.3: barbitúricos, excluindo tiobarbitúricos; T42.4: benzodiazepínicos; T42.6: outras drogas antiepiléticas e sedativos hipnóticos; T42.7: antiepiléticas e sedativos hipnóticos não especificados; T43.0: antidepressivos tricíclicos e tetracíclicos; T43.2: outros antidepressivos e os não especificados; T43.4: neurolépticos do tipo butirofenona e tioxanteno; T43.5: outros antipsicóticos e neurolépticos e os não especificados; T43.8: outras drogas psicotrópicas não especificadas em outra parte; T43.9: droga psicotrópica não especificada; T45.5: anticoagulantes; T46.0: glicosídeos estimulantes do coração e substâncias de ação semelhante; T47: substâncias que atuam primariamente sobre o aparelho gastrointestinal; T50: diuréticos e outras drogas, medicamentos e substâncias biológicas e as não T50: cificas; T50.9: outras drogas, medicamentos e substâncias biológicas as não

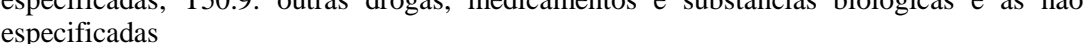

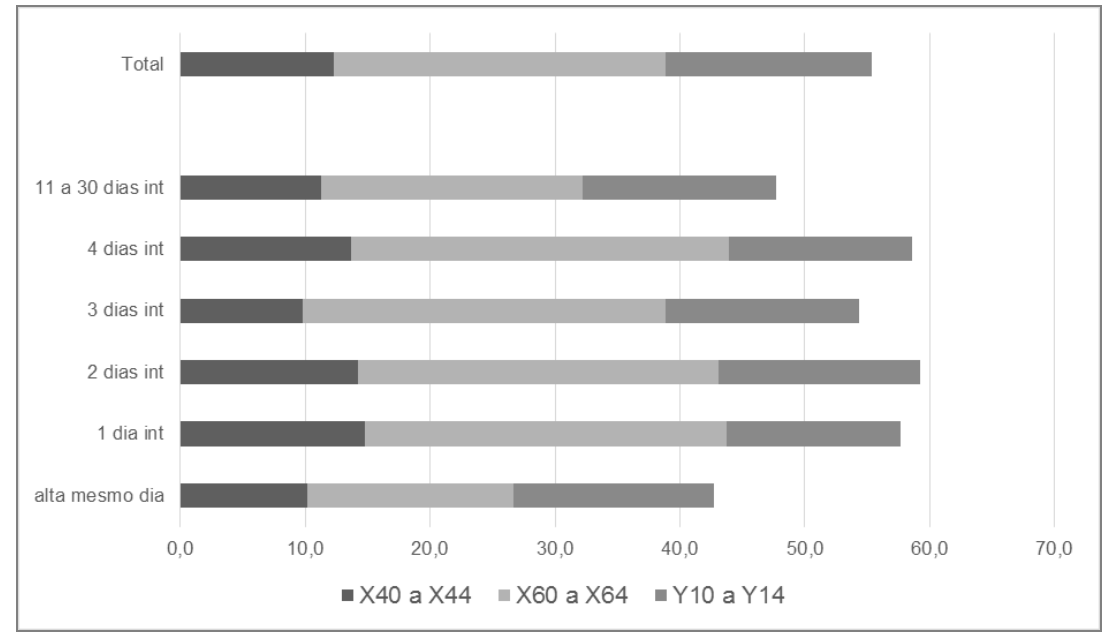

*Fonte: Sistema de Informações Hospitalares

***Nota:

Categoria "alta mesmo dia" referente à soma dos casos de alta/saída no mesmo dia da internação (157 casos) e casos sem data de alta/saída (49 casos)

Códigos da CID-10 - X40 a X44: intoxicações acidentais; X60 a X64: intoxicaçoes intencionais; Y10 a Y14: intoxicações cuja causa não foi determinada

Figura 4: Distribuição das internações por intoxicação medicamentosa classificadas segundo intenção e tempo de internação: município de São Paulo - 2004 a 2006.

\section{DISCUSSÃO}

$\mathrm{O}$ emprego da $\mathrm{AIH}$, um documento de faturamento da unidade hospitalar ao órgão financiador, poderia comprometer a validade dos dados em estudos epidemiológicos ${ }^{10}$. No entanto, em estudos de internações associadas a procedimentos de custos variados os erros ou fraudes não produziriam erros diferenciais, não resultando em tendências ou vieses importanteguras ${ }^{10}$. Foi possível verificar que a proporção de internações por intoxicação medicamentosa cresceu no período de estudo, sendo $0,14 \%$ do total de internações do município em 2004, 0,16\% em 2005 e $0,19 \%$ em 2006.

A incorporação de informações sobre o uso de medicamentos no SIH/SUS viabilizaria a condução de estudos de farmacovigilância a partir de dados secundários ${ }^{10}$. Sugere-se a adoção da classificação Anatomical Therapeutic Clinical (ATC), habitualmente utilizada em estudos sobre a utilização de medicamentos.

Verificou-se predomínio do sexo feminino para o banco de dados de internações no município e para as internações selecionadas para o estudo. No entanto, observouse predomínio de internações em pacientes com idade até 36 anos $(50,1 \%$ dos casos) no banco de dados referente às internações, enquanto que $49,8 \%$ dos casos de internação por intoxicação medicamentosa ocorreram para os indivíduos com 20 a 49 anos (a idade média das pessoas internadas foi de 33,5 anos). Essas diferenças podem ser atribuídas ao fato de a faixa etária predominante para as internações por intoxicação corresponder à mais suscetível a tentativas de suicídio. Observou-se que a contribuição de intoxicações intencionais é a mais expressiva (Figura 4). O suicídio continua sendo considerado um problema social e de saúde pública, sendo que os jovens são mais vulneráveis ao comportamento suicida $^{8}$, embora os idosos também apresentem risco aumentado para comportamento suicida em muitos países ${ }^{9}$.

Além do predomínio de indivíduos mais jovens entre os casos estudados, verificamos, também, que houve aumento dos coeficientes de internação para a faixa etária de 20 a 49 anos para o período de estudo. Estudos mostram o predomínio de pacientes jovens hospitalizados por intoxicação medicamentosa ${ }^{10}$.

Por outro lado, observou-se maiores coeficientes de internação para os mais idosos (idade igual ou superior a 70 anos) para todo o período estudado. No Brasil, a população idosa não costuma ser prioridade nos estudos sobre causas externas, devido ao predomínio dos casos entre os jovens ${ }^{11}$.

A população idosa brasileira vem crescendo muito rapidamente a partir da segunda metade do século passado, tanto em números absolutos quanto em números relativos. A 
maior prevalência de problemas crônicos de saúde faz dos idosos grandes consumidores de serviços de saúde e de medicamentos ${ }^{12}$. A alta prevalência do uso de medicamentos, a polifarmácia e o aumento progressivo do consumo de medicamentos com a idade, mais expressivo para o sexo feminino, são apontados como fatores que podem predispor os idosos a riscos maiores para agravos relacionados ao uso de medicamentos ${ }^{12-16}$, mesmo sendo verificadas baixas taxas de automedicação entre os idosos ${ }^{12-16}$, pois a maior parte dos medicamentos utilizados por esta população são recomendados por um médico ${ }^{12}$.

Para os idosos, os riscos envolvidos no consumo de medicamentos são maiores, se comparados aos do restante da população $^{12}$. Alterações farmacodinâmicas e farmacocinéticas dos medicamentos em decorrência do processo de envelhecimento tornam esse contingente populacional mais vulnerável a eventos adversos, podendo mesmo as doses terapêuticas habituais produzir efeitos tóxicos ${ }^{12,13,17}$. Outros fatores que contribuem para a elevação do risco associados à utilização de medicamentos são déficits cognitivos ou físicos que dificultam o reconhecimento e memorização dos nomes dos medicamentos e problemas relacionados ao acesso ${ }^{12}$, dosagens inadequadas, interações medicamentosas, associações com doses fixas, uso de fármacos pertencentes a uma mesma classe terapêutica, medicamentos sem o valor terapêutico esperado ${ }^{13}$, a polifarmácia ${ }^{12,15}$, e o uso contínuo de medicamentos ${ }^{15}$. A baixa escolaridade dos idosos também é apontada como um fator de risco diante da complexidade dos esquemas medicamentosos utilizados ${ }^{18}$, assim como fato de os idosos fazerem acompanhamento com mais de um médico $^{18}$. Medidas importantes a serem seguidas diante do paciente idoso são o estímulo ao emprego de medidas não farmacológicas; o acompanhamento, com revisão periódica, do conjunto dos medicamentos e de seus possíveis eventos adversos; a preferência por monodrogas em detrimento das associações em doses fixas; a preferência por fármacos de eficácia comprovada através de evidências científicas; a suspensão do uso, sempre que possível; a verificação da compreensão da prescrição e das orientações farmacológicas ou não farmacológicas; a simplificação dos esquemas de administração; e a atenção aos preços ${ }^{17}$.

Tornar gênero um eixo transversal na investigação em saúde é uma aposta na possibilidade de que a produção de evidências sobre desigualdades em saúde entre mulheres e homens, decorrentes das desigualdades de gênero, seja capaz de impactar em políticas públicas voltadas para a redução de tais desigualdades ${ }^{19}$. Vale ressaltar a importância de estudos que apontem diferenças nos perfis de saúde entre homens e mulheres visando identificar possíveis variações de percepções e práticas relativas ao gênero na compreensão dos fenômenos de saúde-doença ${ }^{19}$.

A observação de que fatores distintos podem estar relacionados à suscetibilidade à intoxicação para diferentes faixas etárias pode ser um motivo para que, dentro de cada faixa etária, o predomínio de gênero seja diferente. Os meninos sofrem intoxicação com maior frequência do que as meninas, em quase todas as regiões do mundo, provavelmente devido a diferenças na socialização - na maior parte das culturas não é estimulada a adoção de comportamentos de risco para as meninas, assim como seu envolvimento em atividades ao ar livre ou sem supervisão ${ }^{20}$. No presente estudo, os coeficientes de internação por intoxicação medicamentosa para os menores de 4 anos foram maiores para o gênero masculino. Já entre os idosos, observou-se predomínio das intoxicações para o gênero feminino. A maior prevalência de utilização de medicamentos pelas mulheres pode estar relacionada a este fato ${ }^{12,16}$. Alguns dos aspectos que determinam a maior utilização de medicamentos pelas mulheres são a maior consciência em relação às necessidades de saúde, sua atuação no cuidado com a saúde dos membros da família, busca mais frequente e precoce de serviços de saúde, provavelmente devido a maiores oportunidades de cuidado $^{21}$.

Verificou-se um predomínio das intoxicações intencionais (tentativas de suicídio) entre as internações estudadas. No entanto, um grande número de intoxicações foi classificado como intoxicações com intenção não determinada. Sua adequada atribuição poderia trazer resultados diferentes em relação à frequência e à magnitude das internações por intoxicação medicamentosa acidental e intencional.

Em estudo que analisou a morbimortalidade por causas externas em indivíduos com 60 anos e mais no Brasil, para o ano de 2000, observou-se que $29,2 \%$ dos casos estudados corresponderam à categoria exposição acidental a fatores não especificados. Os autores consideraram que tal fato não pode ser justificável entre as hospitalizações, pois existe a oportunidade para o esclarecimento do tipo de causa. Ainda segundo os autores do estudo, o resultado constitui uma limitação e aponta para a necessidade de melhorar a qualidade dessa informação ${ }^{11}$. O mesmo pode ser dito para este estudo, que encontrou, além dos altos percentuais de internações atribuídas a intoxicação com intenção não determinada, uma grande proporção de intoxicações cujo agente não foi esclarecido (código T50.9: outras drogas, medicamentos e substâncias biológicas e as não especificadas). Isso pode ser indício de falta de informação sobre o medicamento no prontuário médico (não especificados) ou falta de detalhamento adequado para a codificação da CID-10 para sua caracterização (outras drogas).

Os benzodiazepínicos corresponderam a $7,5 \%$ das internações, ficando em segundo lugar entre as substâncias observadas no diagnóstico principal da internação. São medicamentos sob controle especial; $o$ fato de tais medicamentos só poderem ser utilizados quando prescritos por profissional de saúde, por estarem sujeitos a dispensação com a retenção de receita ou da notificação de receita, deveria ser suficiente para garantir a segurança na utilização, ao menos na maioria dos casos. Outros autores também observaram em estudo transversal conduzido na cidade de Maringá, no Paraná, que os medicamentos envolvidos nas intoxicações com maior frequência foram os que agem no sistema nervoso central $(57,2 \%)$, com predomínio dos que estão sob controle de prescrição ${ }^{7}$. O uso de medicamento cuja prescrição é controlada foi observado em $66,2 \%$ dos casos intencionais. Levando em conta esses resultados, os autores salientam a necessidade de treinamento da equipe de saúde para a condução de orientação quanto ao uso correto e estocagem adequada, assim como de um trabalho multidisciplinar para a prevenção, detecção, tratamento e notificação das intoxicações. Em outro estudo ficou demonstrado que o uso na vida de benzodiazepínicos na população das 107 maiores cidades do Brasil, para os habitantes entre 12 e 65 anos de idade, foi de 3,3\%, porcentagem considerada pelos autores semelhante à observada nos EUA $(5,8 \%)^{22}$.

Os antibióticos sistêmicos não especificados (T36.9) foram responsáveis por $7 \%$ das internações e ficaram em terceiro lugar entre os medicamentos mais implicados nas intoxicações. Tais medicamentos sempre apresentaram a tarja contendo os dizeres: "Venda sob prescrição médica", mas passaram a ser vendidos com a exigência de apresentação de receituário e retenção de cópia do mesmo apenas em 2011. Os casos selecionados para o presente estudo podem estar sujeitos a maior influência dos efeitos da automedicação devido a não estarem sujeitos a apresentação e cópia de prescrição. Novos estudos devem ser realizados para avaliar o impacto dessa legislação nas intoxicações e eventos $\operatorname{adversos}^{23}$.

Foi verificado que há diferença entre os agentes 
envolvidos nas internações com relação ao tempo de internação. As intoxicações agudas por opiáceos (F11.0); sedativos e hipnóticos (F13.0); outros estimulantes, inclusive cafeína (F15.0); alucinógenos (F16,0); e o uso de múltiplas drogas e ao abuso de outras substâncias psicoativas (F19.0) em pacientes com transtornos mentais e comportamentais foram mais longas. Dentre os casos com mais de 100 dias de internação (correspondentes a $0,1 \%$ dos casos estudados) também há quatro casos cuja internação se deve ao uso de múltiplas drogas ou outras substâncias psicoativas (F19.0) (resultados não apresentados). Estes achados sugerem que o abuso e associação de substâncias podem trazer consequências mais graves, o que ajudaria a justificar o tempo maior de internação observado.

O disposto na CID-10 determina que para as condições de morbidade determinadas por causas externas, a natureza da condição (códigos $\mathrm{T}$ selecionados para o presente estudo) e a circunstância (códigos X e Y selecionados para o presente estudo) devem ser codificadas. O código da condição principal a ser adotado deverá descrever a natureza da condição $^{24}$. Alguns dos casos selecionados para o estudo foram codificados sem que fosse observada essa regra.

\section{CONCLUSÃO}

A premissa fundamental da saúde pública é a de que as causas externas podem ser previsíveis e, portanto, evitáveis ${ }^{11}$ A intoxicação é classificada pela CID como uma causa externa. As diferenças observadas nos resultados em números absolutos (predomínio da faixa etária de 20 a 49 anos de idade) e em coeficientes (maiores para os mais idosos) demonstra a importância de se ponderar a relação entre os dados e a população de onde eles provem. Assim, podemos verificar que há necessidade de ações para diminuição do número de casos de intoxicações entre os jovens, o que pesa no total de internações por intoxicações estudadas, mas que a relevância das mesmas na população idosa também traz a necessidade de busca de soluções.

\section{AGRADECIMENTOS}

SIH - DATASUS: pela disponibilização dos dados utilizados no estudo

\section{FINANCIAMENTO}

CAPES. O presente artigo é parte da tese de Janessa de Fátima Morgado de Oliveira, apresentada ao Programa de Pós-Graduação em Saúde Pública da Faculdade de Saúde Pública da Universidade de São Paulo para obtenção do título de Doutora em Ciências, defendida em 3 fevereiro de 2017.

\section{REFERÊNCIAS}

1. Romano-Lieber NS. Farmacoepidemiologia. In: Cordeiro BC, Leite SN. O Farmacêutico na Atenção à Saúde. Univali. São Paulo, 2005.

2. Mota DM, Melo JRR, Freitas DRC, Machado M. Perfil de mortalidade por intoxicação com medicamentos no Brasil, 1996-2005: retrato de uma década. Ciênc saúde coletiva. 2012; 17(1):61-70

3. OPAS. Avaliação da assistência farmacêutica no Brasil: estrutura, processo e resultados. Brasília (DF): Organização Panamericana de Saúde, Ministério da Saúde, 2005.

4. Silvério MS, Leite ICG. Qualidade das prescrições em município de Minas Gerais: uma abordagem farmacoepidemiológica. Rev Assoc Med Bras. 2010; 56(6):675-80.

5. WHO. How to investigate drug use in health facilities selected use drug indicators. Action Programme on Essential Drugs. Genebra, 1993.
6. Santos SA, Legay LF, Aguiar FP, Lovis GM, Abelha L, Oliveira SP. Tentativas e suicídio por intoxicação exógena no Rio de Janeiro, Brasil: análise das informações através de linkage probabilístico. Cad Saúde Pública. 2014; 30(5):1057-66.

7. Margonato FB, Thomson Z, Paoliello MMB. Acute intentional and accidental poisoning with medications in a southern Brazilian city. Cad Saúde Pública. 2008; 25(4):849-56.

8. Bernardes SS, Turini CA, Matsuo T. Perfil das tentativas de suicídio por sobredose intencional de medicamentos atendidas por um Centro de Intoxicações do Paraná, Brasil. Cad Saúde Pública. 2010; 26(7):1366-72.

9. WHO. Public Health action for the prevention of suicide - a framework. Genebra, 2012.

10. Rozenfeld S. Agravos provocados por medicamentos em hospitais do Estado do Rio de Janeiro, Brasil. Rev Saúde Pública. 2007; 41(1)108-15.

11. Gawryszewski VP, Jorge MHPM, Koizumi MS. Mortes e internações por causas externas entre os idosos no Brasil: o desafio de integrar a saúde coletiva e atenção individual. Rev Assoc Med Bras. 2004; 50(1):97-103.

12. Loyola Filho AI, Uchoa E, Lima-Costa MF. Estudo epidemiológico de base populacional sobre consumo de medicamentos entre idosos na Região Metropolitana de Belo Horizonte, Minas Gerais, Brasil. Cad Saúde Pública. 2006; 22(12): 2657-67.

13. Flores VB, Benvegnú LA. Perfil de utilização de medicamentos em idosos da zona urbana de Santa Rosa, Rio Grande do Sul, Brasil. Cad Saúde Pública. 2008; 24(6): 1439-46.

14. Flores LM, Mengue SS. Uso de medicamentos por idosos em região do sul do Brasil. Rev Saúde Pública. 2005; 39(6):924-9.

15. Pizzol TSD, Pons ES, Hugo FN, Bozzetti MC, Souza MLR, Hilgert JB. Uso de medicamentos entre idosos residentes em áreas urbanas e rurais de município no Sul do Brasil: um estudo de base populacional. Cad Saúde Pública. 2012; 28(1):104-14.

16. Ribeiro AQ, Rozenfeld S, Klein CH, César CC, Acurcio FA. Inquérito sobre uso de medicamentos por idosos aposentados, Belo Horizonte, MG. Rev Saúde Pública. 2008; 42(4):724-32.

17. Rozenfeld S. Prevalência, fatores associados e mau uso de medicamentos entre idosos: uma revisão. Cad Saúde Pública. 2003; 19(3):717-24.

18. Marin MJS, Cecílio LCO, Perez AEWUF, Santella F, Silva CBA, Gonçalves Filho JR et al. Caracterização do uso de medicamentos entre idosos de uma unidade do Programa Saúde da Família. Cad Saúde Pública. 2008; 24(7):1545-55.

19. Villela W, Monteiro S, Vargas E. A incorporação de novos temas e saberes nos estudos em saúde coletiva: o caso do uso da categoria gênero. Ciênc saúde coletiva. 2009; 14(4):997-1006.

20. WHO. World report on child injury prevention. WHO Library Cataloguing-in-Publication. Genebra, 2008.

21. Francisco PMSB, Bastos TF, Costa KS, Prado MAMB, Barros MBA. The use of medication and associated factors among adults living in Campinas, São Paulo, Brazil: differences between men and women. Ciênc saúde coletiva. 2014; 19(12):4909-21

22. Galduróz JCF, Noto AR, Nappo AS, Carlini EA. Uso de drogas psicotrópicas no Brasil: pesquisa domiciliar envolvendo as 107 maiores cidades do país - 2001. Rev Latino-am Enfermagem. 2005; 13(número especial): 888-95.

23. Paula TC, Bochner R, Montilla DER. Análise clínica e epidemiológica das internações hospitalares de idosos 
decorrentes de intoxicações e efeitos adversos de medicamentos, Brasil, de 2004 a 2008. Ver Bras Epidemiol. 2012; 15(4):828-44

24. WHO. International Statistical Classification of Diseases and Related Health Problems. $10^{\text {th }}$ Revision. Volume 2: Instruction Manual. 2010 ed. Genebra, 2011.

\section{CONFLITO DE INTERESSES}

Os autores declaram não haver conflitos de interesse.

\section{AUTOR PARA CORRESPONDÊNCIA}

Janessa de Fátima Morgado de Oliveira

janessa@usp.br

Submetido em 29/03/2018

Aceito em 11/05/2018 